Notfall Rettungsmed 2017 $\cdot 20: 501-508$

DOI 10.1007/s10049-017-0276-8

Online publiziert: 15 . Februar 2017

(c) Der/die Autor(en) 2017. Dieser Artikel ist

eine Open-Access-Publikation.

CrossMark

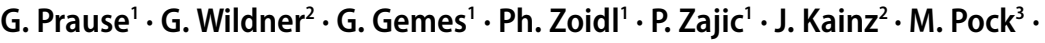 \\ H. Trimmel ${ }^{4}$ \\ 'Klinische Abteilung für Allgemeine Anästhesiologie, Notfall- und Intensivmedizin, Univ. Klinik für \\ Anästhesiologie und Intensivmedizin, LKH Univ. Klinikum Graz, Graz, Österreich \\ ${ }^{2}$ Abteilung für Anästhesiologie und Intensivmedizin, LKH Hochsteiermark-Standort Bruck/Mur, \\ Bruck/Mur, Österreich \\ ${ }^{3}$ Medizinercorps des Roten Kreuzes Graz-Stadt, Graz, Österreich \\ ${ }^{4}$ Abteilung für Anästhesie, Notfall- und Allgemeine Intensivmedizin, KH Wr. Neustadt, Wr. Neustadt, \\ Österreich
}

\title{
Abgestufte präklinische Notfallversorgung - Modell Graz
}

medizinisch attraktiver gestaltet werden kann.

Die Notfallversorgung in Österreich basiert im Wesentlichen auf zwei Säulen: das überwiegend aus ehrenamtlichen Mitarbeitern des nichtärztlichen Rettungsdienstes bestehende System der Rettungs- und Notfallsanitäter und dem - überwiegend krankenhausgestützten - Notarztsystem. Überschreitet der Schweregrad eines Notfallgeschehens den Kompetenzbereich des Rettungspersonals, wird als zweite Instanz ein Notarzt hinzugezogen. Während der von den Rettungsorganisationen gestellte Sanitätsdienst als durchaus stabiler Faktor angesehen werden kann, wird die flächendeckende Vorhaltung von Notärzten schwieriger, sodass mancherorts Notarztdienste bereits nicht mehr besetzt werden können. Diesen Notärztemangel nur mit den Erfordernissen des gemäß EU-Vorschrift verschärften Arbeitszeitgesetzes und einer eher unattraktiven Entlohnung zu begründen, trifft nur einen Teil des Problems. Ein wesentlicher Faktor ist das schwindende Interesse der Kollegen an der Notfallmedizin, verursacht durch ein unattraktives Arbeitsumfeld. Da auch andere Organisationsmodelle hier für die Zukunft mögliche Lösungsansätze bieten könnten, soll dieses Konzept einer abgestuften Notfallversorgung vorgestellt und diskutiert werden. Am Beispiel des Notarztsystems Graz wird gezeigt, dass durch eine Strukturanpassung im Rettungsdienst auch die Tätigkeit der Notärzte vor allem

\section{Zur aktuellen Situation}

Die Ausbildung der Rettungssanitäter (RS) umfasst in Österreich $260 \mathrm{~h}$, bestehend aus $100 \mathrm{~h}$ theoretischem Unterricht und 160 h aktiver Diensttätigkeit im Rettungsdienst unter Supervision. Im Vergleich zu anderen europäischen Ländern (- Tab. 1) ist dies relativ wenig, berücksichtigt aber den Umstand, dass etwa $90 \%$ des Rettungsdienstes, insbesondere außerhalb der Bundeshauptstadt Wien, von ehrenamtlichem Personal bzw. Wehr- oder Zivildienstleistenden getragen wird. Eine Ausbildung im Krankenhaus ist aktuell für diese Helfer nicht vorgesehen; dies erfolgt erst in der Stufe der Notfallsanitäter. Die rechtlich zulässigen rettungstechnischen Fertigkeiten des RS bestehen im Wesentlichen in den Maßnahmen der „Erweiterten Ersten Hilfe" und beinhalten die Bedienung eines (S)AED (semiautomatischer Defibrillators; [12]). Eine Ausbildung zum Sanitäter mit höheren Kompetenzen wie im österreichischen Sanitätergesetz [12] grundsätzlich vorgesehen (Notfallsanitäter bzw. Notfallsanitäterin bis zur höchsten Notfallkompetenzstufe Intubation (NKI) und einem Umfang von etwa $1400 \mathrm{~h}$ ) - werden zwar mancherorts absolviert, aber in der Praxis dann außerhalb des Dienstes am Notarztwagen kaum umgesetzt. In organisierter Form gibt es dies aktuell nur bei der Wiener
Berufsrettung bzw. im Rettungs- und Notarztdienst Graz. Bei letzterem wird das Modell einer abgestuften Versorgung seit mehr als 30 Jahren praktiziert. Diese Notfallsanitäter (NKI) besetzen eigene Notfallrettungswägen („Jumbo's“), welche als Zwischenstufe im Leitstellensystem geführt und auch nach eigenen Indikationslisten zum Notfallort entsandt werden. Der Einfluss eines derartigen 3-stufigen Systems auf die notärztliche Einsatzaktivität soll im vorliegenden Beitrag anhand einer vergleichenden Darstellung der Einsatzstatistik zweier systemtypischer, bodengebundener Notarztsysteme - Graz und Wiener Neustadt - vorgestellt werden.

\section{Notarztdienst}

Das moderne Notarztwesen wurde in Österreich vor mehr als 30 Jahren schrittweise etabliert, um möglichst frühzeitig lebensrettende ärztliche Maßnahmen bereits vor Ort einleiten zu können. Im Sinne eines "vorverlagerten Schockraums“ wurden die meisten Systeme an Krankenhäuser angegliedert und mit Klinikärzten besetzt. Wie mit mehreren Studien belegbar $[4,9,15]$, sind die Einsatzzahlen in den letzten Jahren deutlich gestiegen. Die Kernaufgabe, nämlich die Versorgung von kritisch erkrankten bzw. verletzten Patienten, macht mittlerweile nur mehr einen geringen Prozentsatz $(<10 \%)$ aller Anforderungen aus. In einer Vielzahl von Fällen wird der Notarzt als Ersatz für fehlende medizini- 
Tab. 1 Ausbildungsstunden für Sanitäter in den Ländern Deutschland, Österreich und der Schweiz

\begin{tabular}{lllll} 
& Deutschland & Österreich & Schweiz \\
\hline $\begin{array}{l}\text { Basic Level } \\
\text { Theorie }\end{array}$ & Rettungshelfer & Rettungssanitäter(RS) & Transporthelfer \\
$\begin{array}{l}\text { Praxis (KH) } \\
\text { Summe }\end{array}$ & 160 & 100 & 25 \\
\hline $\begin{array}{l}\text { Advanced Level } \\
\text { Theorie }\end{array}$ & $30(80)$ & 160 & 25 \\
\hline $\begin{array}{l}\text { Praxis (KH) } \\
\text { Summe }\end{array}$ & 200 & 260 & 50 \\
\hline $\begin{array}{l}\text { Expert Level } \\
\text { Theorie }\end{array}$ & $160(160)$ & $\begin{array}{l}\text { Notfallsanitäter (NKV) } \\
\text { (Aufbauend auf RS) }\end{array}$ & Transportsanitäter \\
\hline $\begin{array}{l}\text { Praxis (KH) } \\
\text { Notfallsanitäter }\end{array}$ & 170 & 680 \\
\hline $\begin{array}{l}\text { Summe } \\
\text { Rettungsmediziner } \\
\text { Graz }\end{array}$ & $\mathbf{5 2 0}$ & $360(80)$ & $1120(320)$ \\
\hline
\end{tabular}

sche Strukturen (Hausarzt, kassenärztlicher Bereitschaftsdienst) herangezogen, aber auch Nachforderungen durch den (vermeintlich oder tatsächlich) überforderten Rettungsdienst haben deutlich zugenommen.

Diese Entwicklung ist mit Sicherheit auch ein wesentlicher Faktor, warum vor allem erfahrene Kollegen für den Notarztdienst nicht (mehr) zur Verfügung stehen wollen. Aus Sicht der Aufgabenstellung einer evidenzbasierten und effektiven Notfallmedizin braucht es jedoch qualifizierte Ärzte mit breitem Erfahrungsschatz, die auch unter den schwierigen Bedingungen des präklinischen Einsatzes rasch und richtig agieren können. Ein Mangel an derart erfahrenen Notärzten ist mittlerweile in Österreich jedoch offensichtlich.

\section{Medizinercorps Graz}

Schon im Jahre 1890 wurden in Graz infolge des Ärztemangels Medizinstudierende im Rettungsdienst eingesetzt [10]. Diese Tradition konnte bis zum heutigen Tag erhalten und konform zur medizinischen Entwicklung und den gesetzlichen Vorgaben adaptiert werden. Mit Einrichtung des Notarztwesens rückte das Medizinercorps etwas in den Hintergrund, unterstützte die Notärzte aber weiterhin als hochqualifizierte Helfer bzw. agierte als „Vorhut“ bei unklaren Notfallanforderungen. Mit der Einführung der Notfallkompetenz (s. oben Sanitätergesetz 2002) nach Verständigung eines Arztes/Notarztes wurden definierte notfallmedizinische Maßnahmen ermöglicht, die auf Einschätzung der Vitalfunktionen und der Applikation von freigegebenen Arzneimitteln fokussierten [12]. Daraus resultiert, dass viele Einsätze heute auch ohne Anwesenheit des Notarztes durch den sog. „Rettungsmediziner", einem Notfallsanitäter mit Kompetenz Intubation (NKI) abgewickelt werden können. Die Ausbildung der Rettungsmediziner wurde 1983 strukturiert und konsekutiv an die laufenden Änderungen der Ausbildungs-Curricula (Studium Humanmedizin und auch Sanitäterausbildung) angepasst. Neben der Sanitätshilfe werden die im Laufe des Medizinstudiums erworbenen Kompetenzen angerechnet, womit sich eine Mindestausbildungsdauer von mehr als 3000 h ergibt (• Tab. 2). Etwa die Hälfte der Ausbildung $(1500 \mathrm{~h})$ ist die praktische Tätigkeit als Anwärter unter Supervision am Notfallwagen. Dies bedingt, dass neben dem Fahrer und einem Rettungssanitäter immer ein zusätzlicher Helfer zur Verfügung steht (4 Personen). Der Notfallwagen ist ein voll ausgestatteter
Tab. 2 Ausbildung des Medizinercorps

\begin{tabular}{|c|c|}
\hline Rettungsdienst & Stunder \\
\hline Sanitäterausbildung (Basis) & 260 \\
\hline Notfallsanitäter & 480 \\
\hline Kompetenz Venöser Zugang & 50 \\
\hline $\begin{array}{l}\text { Kompetenz Medikamentenappli- } \\
\text { kation }\end{array}$ & 40 \\
\hline Kompetenz Intubation & 110 \\
\hline Phantomübungen & 50 \\
\hline \multicolumn{2}{|l|}{ Studium Humanmedizin } \\
\hline Notfall- und Rettungsmedizin & 90 \\
\hline $\begin{array}{l}\text { „Problem-based learning“, Fallbe- } \\
\text { richte in der Notfallmedizin }\end{array}$ & 30 \\
\hline EKG & 15 \\
\hline \multicolumn{2}{|l|}{ Famulaturen, Praxis und Prüfung } \\
\hline $\begin{array}{l}\text { Anästhesie ( } 4 \text { Wochen plus } 3 \text { Wo- } \\
\text { chen Kinderanästhesie) }\end{array}$ & 280 \\
\hline Innere Medizin (4 Wochen) & 160 \\
\hline Chirurgie (2 Wochen) & 80 \\
\hline $\begin{array}{l}\text { Begleitfahrten unter Supervision } \\
\text { als Rettungsmediziner-Anwärter }\end{array}$ & 1500 \\
\hline $\begin{array}{l}\text { Abschlussgespräch, praktische } \\
\text { Prüfung }\end{array}$ & 15 \\
\hline Gesamtstunden & 3160 \\
\hline
\end{tabular}

Rettungswagen nach der Norm DIN EN 1789 entsprechend einer MICU (Mobile Intensive Care Unit).

\section{3-Stufen-Modell Graz}

Seit den frühen 1980er Jahren werden Stadtgebiet und Umland von Graz ( 400.000 Einwohner im Versorgungsbereich) mit dem abgestuften Notfallsystem versorgt. Neben den beiden arztbesetzten Notarzteinsatzfahrzeugen (NEF) der öffentlichen Krankenhäuser (LKH Universitätsklinikum Graz bzw. UKH Graz/LKH Graz Südwest, Standort West) gibt es zwei sog. Notfallrettungswägen („Jumbo“) des Grazer Roten Kreuzes, die vom Medizinercorps betreut werden (s. oben) [10]. Die Rettungsmediziner (s. oben) werden bei nicht eindeutigem, jedoch möglicherweise kritischem Notfallgeschehen (z. B. Kollaps, fragliche Bewusstlosigkeit, Sturzgeschehen usw.) zum Notfallort entsendet und stellen damit nach den Rettungssanitätern die zweite Instanz in der Versorgungskette dar. Bei deutlicherem Hinweis auf Vorliegen einer Vitalgefährdung wird zeitgleich auch ein Notarztmittel zum Einsatzort beordert, sodass in diesen Fällen zu- 
Notfall Rettungsmed 2017 $\cdot 20: 501-508$ DOI 10.1007/s10049-017-0276-8

(c) Der/die Autor(en) 2017. Dieser Artikel ist eine Open-Access-Publikation.

G. Prause · G. Wildner · G. Gemes · P. Zoidl · P. Zajic · J. Kainz · M. Pock · H. Trimmel

Abgestufte präklinische Notfallversorgung - Modell Graz

\section{Zusammenfassung}

Hintergrund. Die präklinische Notfallversorgung in Österreich stützt sich im Regelfall auf ein 2-stufiges System. Liegen keine erkennbaren Störungen einer Vitalfunktion vor, werden als primäre Rettungskräfte zum überwiegenden Teil ehrenamtlich tätige Rettungssanitäter mit einer Ausbildung von zumindest $260 \mathrm{~h}$ zum Notfallort entsandt. Überschreitet die Notfallsituation deren Kompetenz, stellt die Nachalarmierung von Notärzten die nächste (und zugleich ultimativ höchste) Stufe der Regelversorgung dar. In der letzten Dekade ist allerdings österreichweit der Eindruck einer steigenden Anzahl an Notarztalarmierungen zu bemerken. Die Anzahl notwendiger Interventionen der Notärzte ist demgegenüber vergleichsweise niedrig. Im Notarztsystem der steirischen Landeshauptstadt Graz existiert seit 30 Jahren ein 3-stufiges System: Hier ergänzt das "Medizinercorps" des Roten Kreuzes als zwischengeschaltete Einrichtung den Ret- tungs- bzw. Notarztdienst. Diese Einrichtung bewältigt mit Medizinstudenten, die als Notfallsanitäter der höchsten Kompetenzstufe ausgebildet sind, auch Notfälle, für die anderenorts der Notarzt ausrücken müsste. Methodik. Auf Basis von Daten des Statistischen Zentralamts und den Rettungsleitstellen der Bundesländer wurden die Einsatzzahlen der Notarztsysteme in Österreich ermittelt und mit der Bevölkerungszahl korreliert. Darüber hinaus wurden Daten zu notärztlichen Maßnahmen zweier Notarztsysteme - NAW des LK Wiener Neustadt als zwei- und NEF des LKH Universitätsklinikum Graz als 3-stufiges System - analysiert und jeweils mit der Bevölkerungszahl in Relation gesetzt. Ergebnisse. Die Einsatzfrequenzen der Notarztsysteme sind in den österreichischen Bundesländern durchaus unterschiedlich und reichen von 14,9 bis zu 29,7 Einsätzen pro Jahr und 1000 Einwohner (EW). Die Frequenz spezifischer notärztlicher Maßnahmen in Wiener Neustadt und Graz sind bei hochspezifischen Tätigkeiten wie Intubation, Narkoseeinleitung, Katecholamin- und Antiarrhythmikatherapie vergleichbar, notfallmedizinische "Basismaßnahmen" (venöser Zugang, Infusionstherapie mit kristalloiden Lösungen) sind in Graz jedoch signifikant seltener, ebenso wie Einsatzstornierungen und Fehleinsätze.

Schlussfolgerung. Das 3-stufige Grazer Notarztsystem zeigt, dass ein derartiges Modell der größeren Differenzierung notfallmedizinischer Strukturen erlaubt, vorhandene Ressourcen effizienter einzusetzen. Dadurch kommt man dem Ziel näher, allen Notfallpatienten adäquate Hilfe anbieten zu können, ohne das System über Gebühr zu beanspruchen.

Schlüsselwörter

Notarztsystem · Qualität · Sanitäter .

Ausbildung $\cdot$ Notfallsanitäter

\section{Tiered prehospital emergency care-the Graz model}

\section{Abstract}

Abstract. In Austria, prehospital emergency medical service is provided in two tiers. If there are no obvious disturbances of the patient's vital functions, a standard ambulance staffed with predominant voluntary emergency technicians (= EMT, $260 \mathrm{~h}$ of training) will respond to the call. In case the emergency situation exceeds the EMT's level of competence, an emergency physician as next and utmost level of care is summoned to the scene. In Graz, a different model is used consisting of three levels of emergency care. Between basic ambulances and emergency physicians, there is an intermediate step of specialized ambulance staff: medical students as highly qualified emergency technicians, who have completed extended training in emergency medicine of more than $2500 \mathrm{~h}$.
Methods. Demographical data were gathered from the governmental statistical registry and were related to the frequency of emergency calls obtained from the dispatch centers. Specific data about emergency on-scene procedures were evaluated from the emergency systems of Wiener Neustadt as an example of a two-level system and from the emergency system in Graz being representative for the three-level model.

Results. The frequency of emergency physician assignments varies across the federal states of Austria from 14.9 up to 29.7 calls per 1000 inhabitants and year. Specialized measures of emergency medical care like intubation, anesthesia induction, application of catecholamines, and/or antiarrhythmics were comparable in Graz and
Wiener Neustadt. Basic emergency medical techniques and especially false alarms or cancellations were significantly less frequent in Graz.

Conclusions. The three-tier emergency system of Graz enables a judicious and efficient use of emergency care resources, which allows the provision of adequate care for every emergency patient without excessively straining the emergency care system.

Keywords Emergency medical services · Quality control . Emergency medical technicians - Education, medical · Paramedics · Resue paramedics sammen mit dem Notarzt 6 ausgebildete Notfallhelfer zur Verfügung stehen.

\section{Methodik}

Unter Zuhilfenahme von Daten des statistischen Zentralamts wurden die aktuelle Einwohnerzahl eines Bundeslandes mit der Tourismusintensität (Summe der Nächtigungen), heruntergebrochen auf den Tag, addiert und daraus der sog. „Einwohnergleichwert" ermittelt. Die Zahlen über die Notarztmittel wurden durch persönliche Befragung der Notfallreferenten der Landesärztekammern sowie den zentralen Leitstellen erhoben. Zur Berech- nung der Notarztverfügbarkeit in Bezug auf die Bevölkerungszahl wurden nur die bodengebundenen Notarztsysteme eingeschlossen, da die Notarzthubschrauber einerseits eingeschränkte Betriebszeiten haben bzw. andererseits teilweise - in den Tourismusregionen - nur für wenige Monate im Jahr zur Verfügung 
Tab. 3 Notarztdichte in Österreich unter besonderer Hervorhebung von Graz und Wiener Neustadt ( ${ }^{*}$ Einwohnerkennzahl = Summe aus Einwohnerzahl und der durchschnittlichen Nächtigungen pro Tag)

\begin{tabular}{|l|l|l|l|l|l|l|}
\hline Bundesland & Einwohner & $\begin{array}{l}\text { Nächti- } \\
\text { gungen/d }\end{array}$ & $\begin{array}{l}\text { Einwohner- } \\
\text { Kennzahl* }\end{array}$ & $\begin{array}{l}\text { Anzahl } \\
\text { NAW/NEF }\end{array}$ & $\begin{array}{l}\text { Anzahl } \\
\text { RTH (Sai- } \\
\text { sonal } \\
\text { plus) }\end{array}$ & $\begin{array}{l}\text { 1 Notarzt } \\
\text { auf }\end{array}$ \\
\hline Wien & 1.800 .000 & 39.178 & 1.839 .178 & 12 & 1 & 153.264 \\
\hline Niederösterreich & 1.636 .000 & 17.500 & 1.653 .500 & 27 & 3 & 61.240 \\
\hline Oberösterreich & 1.400 .000 & 31.000 & 1.431 .000 & 17 & 5 & 84.176 \\
\hline Steiermark & 1.220 .000 & 31.268 & 1.251 .268 & 21 & 2 & 59.584 \\
\hline Tirol & 730.000 & 120.000 & 850.000 & 20 & $8(8)$ & 42.500 \\
\hline Kärnten & 557.400 & 33.235 & 590.635 & 9 & $2 / 1$ & 65.626 \\
\hline Salzburg & 538.000 & 50.000 & 588.000 & 7 & $3(5)$ & 84.000 \\
\hline Vorarlberg & 378.500 & 14.989 & 393.489 & 6 & $1(1)$ & 65.581 \\
\hline Burgenland & 288.200 & 7816 & 296.016 & 5 & 1 & 59.203 \\
\hline Österreich- & 8.548 .100 & 344.986 & 8.893 .086 & 124 & $26 / 15$ & 71.718 \\
gesamt & & & & & & \\
\hline Graz & 400.000 & 2819 & 402.819 & 2 & 0 & 201.409 \\
\hline Wr. Neustadt & 80.000 & 1000 & 81.000 & 1 & 0 & 81.000 \\
\hline
\end{tabular}

Tab. 4 Auflistung der Notarzteinsätze der Bundesländer für das Jahr 2014 mit besonderer Hervorhebung der Notarztstützpunktes Graz-Klinikum und Wiener Neustadt

\begin{tabular}{l|lll}
\hline Bundesland & Einwohnerkennzahl & Notarzteinsätze & Pro/1000 EW \\
\hline Wien & 1.839 .178 & 54.701 & 29,74 \\
\hline Niederösterreich & 1.653 .500 & 43.936 & 26,83 \\
\hline Oberösterreich & 1.431 .000 & 28.186 & 19,70 \\
\hline Steiermark & 1.251 .268 & 18.740 & 15,00 \\
\hline Tirol & 850.000 & 20.894 & 24,58 \\
\hline Kärnten & 590,635 & 11.958 & 20,25 \\
\hline Salzburg & 588.000 & 9092 & 15,46 \\
\hline Vorarlberg & 393.489 & 7900 & 20,08 \\
\hline Burgenland & 296.016 & 6244 & 21,09 \\
\hline Österreich gesamt & 8.893 .086 & 201.651 & 22,71 \\
\hline Graz - (Klinikum) & 200.000 & 1747 & 8,74 \\
\hline Wr. Neustadt & 80.000 & 2603 & 32,54
\end{tabular}

stehen, während die Notarztwagen ihren Versorgungsbereich 365 Tage und $24 \mathrm{~h}$ abdecken.

Zum Vergleich der notärztlichen Maßnahmen wurden die Daten des Notarztsystems von Wiener Neustadt (Niederösterreich) als Beispiel eines zweistufigen Versorgungssystems mit dem Modell Graz (Steiermark) als dreistufiges System verglichen. Diese Auswahl des zu vergleichenden Notarztsystems wurde aufgrund ähnlicher soziodemographischer Strukturen in einem gemischt städtisch-ländlichen Versorgungsbereich getroffen. Wichtig ist festzustellen, dass sich die Daten des
Modell Graz nur auf den Einzugsbereich des Notarztwagens des Universitätsklinikums ( 200.000 Einwohner, EW) beziehen.

Zur differenzierten Auswertung herangezogen wurden sowohl logistische $\mathrm{Pa}$ rameter des Einsatzablaufs (Storno, Belassung vor Ort, Todesfeststellung) als auch notfallmedizinische Maßnahmen, die eine hohe ärztliche Qualifikation erfordern (Intubation, Narkoseeinleitung, Reanimation) sowie auch jene Tätigkeiten, die auch von Sanitätern ausgeführt gelungen Genüge zu tun, muss in diesen Fällen zwar die Verständigung des Notwerden dürfen. Um den gesetzlichen Re- arztes mit einer Situationsschilderung erfolgen, die physische Anwesenheit des Notarztes am Einsatzort ist aber nicht zwingend. Die Daten wurden unter $\mathrm{Zu}$ hilfenahme von Microsoft Excel 2010 und IBM SPSS Statistics 22 statistisch ausgewertet. Für den Vergleich der Frequenzen der Tätigkeiten zwischen den beiden Stützpunkten wurde die Odds Ratio berechnet und mittels $\chi^{2}$-Test auf Signifikanz überprüft. Es wurden keinerlei direkt patientenbezogene Daten bearbeitet, daher konnte auf ein Votum der Ethikkommission verzichtet werden.

\section{Ergebnisse}

Im Jahr 2014 standen in Österreich 124 Notarztfahrzeuge und 26 Notarzthubschrauber zur Verfügung. • Tab. 3 zeigt die Aufteilung der Notarztmittel sowohl boden- als auch luftgebunden, wobei je nach Saison (Stichwort Wintersportregion) 16 zusätzliche Hubschrauber zur Verfügung stehen; diese sind in der Tabelle in Klammern dargestellt (z. B. Tirol 8(8)). Im Durchschnitt steht somit in Österreich ein Notarzt (in den bodengebundenen Systemen) für etwa $71.700 \mathrm{EW}$ zur Verfügung (• Tab. 3). Die beiden größten Städte haben die im Verhältnis zur Einwohnerzahl geringste Notarztdichte. In Graz-Stadt gibt es für rund $400.000 \mathrm{EW}$,nur“ zwei Notarztsysteme, in Wien kommt auf $150.000 \mathrm{EW}$ ein Notarztmittel. Österreichweit wurden bei einem Einwohnergleichwert von 8.548.000 im Jahr 2014201.651 Notarzteinsätze (boden- und luftgebunden) durchgeführt, woraus sich ein Mittelwert von 23 Einsätzen pro 1000 EW ergibt. In 0 Tab. 4 sind die Verteilung der Einsatzzahlen der einzelnen Bundesländer sowie die Ergebnisse des Notarztsystems Universitätsklinikum Graz und Wiener Neustadt für den speziellen Vergleich gezeigt. In - Abb. 1 wird die Anzahl der Einsätze pro 1000 EW im Jahr 2014 gezeigt. Der österreichische Durchschnitt wird von Wien, Niederösterreich und Tirol überschritten, auffällig deutlich unter dem Durchschnitt liegen die Länder Salzburg und Steiermark. 
Tab.5 Auflistung der Prozentsätze notfallmedizinischer Maßnahmen berechnet auf die Einwohnerzahl (Graz 200.000 vs. Wiener Neustadt 80.000)

\begin{tabular}{|c|c|c|c|c|c|c|c|c|}
\hline & \multicolumn{2}{|l|}{ Graz } & \multicolumn{2}{|c|}{ Wr. Neustadt } & \multirow[b]{2}{*}{$p=$} & \multirow[b]{2}{*}{ OR } & \multicolumn{2}{|c|}{$\begin{array}{l}\text { Konfidenz } \\
\text { Intervall }\end{array}$} \\
\hline & Anzahl & Pro 1000 & Anzahl & Pro 1000 & & & Unteres & Oberes \\
\hline Storno/Leerfahrt & 456 & 2,28 & 623 & 7,849 & $<0,001$ & 3,43 & 3,04 & 3,87 \\
\hline Todesfeststellung & 68 & 0,34 & 45 & 0,56 & 0,008 & 1,65 & 1,14 & 2,41 \\
\hline Reanimation & 108 & 0,54 & 110 & 1,38 & $<0,001$ & 2,55 & 1,95 & 3,32 \\
\hline iv.-Zugang & 892 & 4,46 & 1400 & 17,5 & $<0,001$ & 3,97 & 3,65 & 4,32 \\
\hline $\begin{array}{l}\text { Infusion (als einzige Thera- } \\
\text { pie) }\end{array}$ & 103 & 0,52 & 339 & 4,23 & $<0,001$ & 8,26 & 6,62 & 10,3 \\
\hline Opioidgabe & 194 & 0,97 & 327 & 4,08 & $<0,001$ & 4,23 & 3,54 & 5,05 \\
\hline Sedierung & 166 & 0,83 & 166 & 2,08 & $<0,001$ & 2,5 & 2,01 & 3,1 \\
\hline Ketamine & 58 & 0,29 & 97 & 1,21 & $<0,001$ & 4,16 & 3,02 & 5,79 \\
\hline Glucosegabe & 16 & 0,08 & 29 & 0,36 & $<0,001$ & 4,53 & 2,46 & 8,35 \\
\hline Intubation & 131 & 0,65 & 58 & 0,73 & 0,519 & 1,11 & 0,81 & 1,51 \\
\hline Narkoseeinleitung & 66 & 0,33 & 42 & 0,53 & 0,23 & 1,59 & 1,08 & 2,34 \\
\hline Katecholamine & 27 & 0,14 & 15 & 0,19 & 0,31 & 1,39 & 0,739 & 2,61 \\
\hline Antiarrhythmika & 51 & 0,26 & 31 & 0,39 & 0,65 & 1,52 & 0,97 & 2,38 \\
\hline
\end{tabular}

\section{Medizinische Maßnahmen}

Von den Notarztstützpunkten Wiener Neustadt sowie dem Stützpunkt Universitätsklinikum Graz liegen genaue Daten hinsichtlich der notärztlichen Maßnahmen vor. Bereits die Analyse der logistischen Einsatzdaten zeigt große Unterschiede: In Wiener Neustadt wurden im Jahr 20142602 Notarzteinsätze bei einem Einzugsgebiet von etwa 80.000 EW absolviert, entsprechend 32,5 Einsätzen/1000 EW. Im Notarztsystem des Universitätsklinikums Graz wurden im selben Beobachtungszeitraum bei einem Versorgungsbereich von etwa 200.000 EW (Graz-Ost und Umgebung) nur 1747 Einsätze durchgeführt, woraus 8,7 Einsätze/1000 EW und Jahr resultieren.

Bezieht man sich auf die Einwohnerzahl wird deutlich, dass die Notärzte in Graz signifikant weniger Stornierungen und Todesfeststellungen absolvieren ( 0 Tab. 5). Ebenso sind isoliert durchgeführte notfallmedizinische Basismaßnahmen wie das Legen eines intravenösen Zugangs, Infusionstherapie und Analgosedierung deutlich seltener als in Wiener Neustadt. Andererseits sind notarztspezifische Maßnahmen wie Narkoseeinleitung, Katecholamin- und Antiarrhythmikatherapie in vergleichbarer Häufigkeit anzutreffen. Diese Unterschiede sind anschaulich in $\mathbf{0} \mathbf{A b b} \mathbf{2}$ dargestellt. Die Glucosegabe und die Applikation von Analgetika und Sedativa Maßnahmen, die für Notfallsanitäter im Sinne des Sanitätergesetzes [12] freigegeben sind, machen einen Anteil von etwa 10 Fällen pro $1000 \mathrm{EW}$ aus. Der wohl bedeutendste Unterschied zeigt sich bei jenen Patienten, die vom Notarzt lediglich eine Infusionstherapie ohne zusätzliche Medikation erhielten. Diese Maßnahme wird in Wiener Neustadt im Vergleich zu Graz 8-mal häufiger gesetzt. Die Auflistung der notfallmedizinischen Maßnahmen ist natürlich nur beispielhaft zu sehen und für die Überarbeitung des Portfolios notärztlicher Tätigkeiten unvollständig.

\section{Diskussion}

Die Aufarbeitung der Frequenz notfallmedizinischer Einsätze in Österreich zeigt ein sehr unterschiedliches Bild, welches sich vielerorts nur schwer erklären lässt. So ist die Anforderung eines Notarztes in Wien am häufigsten, gefolgt von Niederösterreich und Tirol. In diesem Land lässt sich die hohe Anforderungsrate evtl. mit der hohen Notarztdichte erklären. Es stehen hier nämlich nicht nur 20 Notarztfahrzeuge, sondern saisonal noch zusätzlich bis zu 16 Notarzthubschrauber zur Verfügung. Damit reduziert sich die Einwohnerzahl, für die ein Notarztmittel zur Verfügung steht von 42.500 auf (saisonal) für 23.600 Menschen, was neben dem hohen Tourismusanteil auch durch die besonderen geografischen Gegebenheiten des alpinen Raums begründbar ist. Sowohl in der Steiermark als auch in Salzburg scheinen die Rettungsleitstellen die Ressource Notarzt deutlich restriktiver einzusetzen. Deren niedrige Einsatzfrequenz wird lediglich durch das Modell der abgestuften Notfallversorgung des Grazer Modells unterboten.

Die Frage, ob Notärzte in der Steiermark und Salzburg zu wenig eingesetzt werden, lässt sich ohne Outcome-Analysen der Notfallpatienten nicht qualifiziert beantworten. Davon ausgehend, dass medizinische Maßnahmen nur bei entsprechender Indikation durchgeführt werden, ist jedoch schon ein Rückschluss auf deren Notwendigkeit möglich. Die Auswertung der notfallmedizinischen Maßnahmen bringt hier doch deutliche Hinweise: Das Ergebnis, dass der Anteil qualifizierter notärztlicher Interventionen (Intubation, Narkose-Einleitung usw.) bezogen auf die Einwohnerzahl gleich ist, obwohl die Einsatzfrequenz in Wiener Neustadt fast um das 4-Fache höher ist, lässt die Interpretation zu, dass der Unterschied nicht die Erkrankungsund Verletzungsschwere ist, sondern dass viele Maßnahmen im abgestuften Grazer System vom nichtärztlichen Rettungspersonal übernommen werden. Damit 


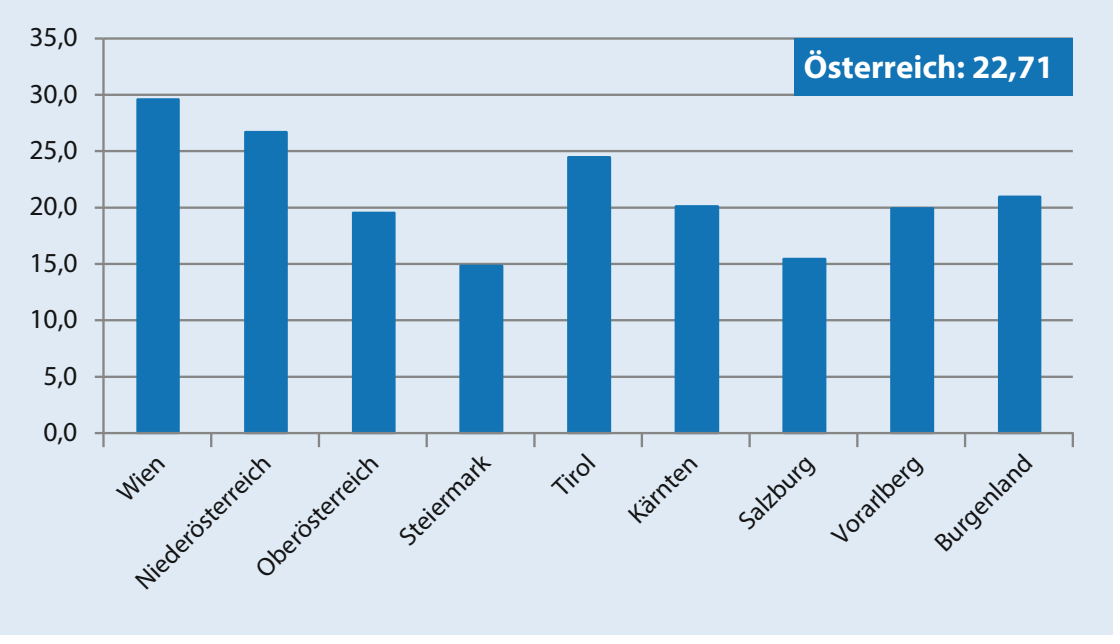

Abb. 1 \& Zahl der Einsätze pro 1000 Einwohner und Jahr aufgeteilt auf die 9 Bundesländer. $t$ tausend EW

kann auch das Argument entkräftet werden, dass durch eine zurückhaltende Alarmierung Notfallpatienten „unterversorgt" sein könnten. Ein abgestuftes System erlaubt im Gegenteil, die Rettungskräfte gemäß ihrer Qualifikationen besser einzusetzen und so letztlich auch eine bessere Verfügbarkeit bedürfnisadäquater Hilfe zu gewährleisten.

Zur Diskussion steht im Notarztdienst auch immer die Frage, inwieweit Analgesie und Sedierung ärztliche Präsenz erfordert. Aufgrund der Datenlage könnte man ableiten, dass diese von Sanitätern selbstständig angewandt würden, was vor allem in Bezug auf die Opioide klar gesetzeswidrig wäre. Demgegenüber muss festgehalten, dass eine präklinische Schmerzbehandlung nicht zwangsläufig und immer medikamentös erfolgen muss, sondern auch durch kompetente sanitätshilfliche Maßnahmen wie Schienung, Lagerung und/oder Zuwendung durchgeführt werden kann. Eine Schmerzbehandlung mit S-Ketamin in Kombination mit Midazolam ist auch in den Arzneimittellisten freigegeben, sodass in vielen Fällen ein Notarzt von der Therapie zwar gesetzeskonform verständigt, aber nicht als zusätzliches Rettungsmittel nachgeholt wird. Opioide werden von den Rettungsmedizinern nicht appliziert.

Vorliegende Arbeit soll weder die Bedeutung der Freiwilligkeit im Rettungsdienst noch den Stellenwert des
Notarztwesens in Frage stellen. ErsteHilfe-Projekte im Sinne der Nachbarschaftshilfe und eines am schnellsten verfügbaren "First-Responder“ sind nicht wegzudenkende Stützen der präklinischen Versorgung. Wie auch immer wiederkehrende Großschadensereignisse und Naturkatastrophen bzw. z. B. auch die Flüchtlingswelle im Jahr 2015 eindrucksvoll gezeigt haben, ist die große Zahl an freiwilligen Sanitätern in Österreich für eine funktionierende Erstversorgung elementar. Nichtsdestotrotz muss festgestellt werden, dass die Sanitäterausbildung in Österreich die im deutschsprachigen Raum mit Abstand geringste Stundenanzahl aufweist [1, $14,16]$ und außerdem noch durch die Tatsache gekennzeichnet ist, dass die rechtlichen Rahmen bei weitem nicht ausgeschöpft werden. Sanitätshilfe im „Expert Status“ wird kaum gelebt und es gibt de facto auch keine „notfallmedizinischen Versorgungen“ durch Sanitäter. Ebenso ist aber auch der Stellenwert von Notärzten als das höchst qualifizierte Glied in der Rettungskette unbestritten und dessen Sinnhaftigkeit mit hoher Evidenz hinterlegt [2, 3].

In der Diskussion von Strategien zur Optimierung der Notfallversorgung wird gerne das angloamerikanische Modell eines „Paramedics-System“ $[6,8]$ dem frankogermanischen „Notarztsystem“ kontradiktorisch gegenübergestellt [11, 13]. Sehr oft werden die beiden Modelle im gegenseitigen Ausschluss betrachtet und auch durchaus emotional diskutiert. Tendenziell zeichnet sich jedoch eine Annäherung der beiden Standpunkte ab: Ursprünglich rein „Paramedicsorientierte" Emergency-Medical-Service(EMS)-Einrichtungen akzeptieren angesichts der aktuellen Datenlage medizinischen Wissens zunehmend den Stellenwert frühzeitiger notärztlicher Maßnahmen [2, 5, 7] und erweiterten die Notfallteams um qualifizierte Ärzte für ausgewählte Indikationen. Diese kommen entweder durch Einsatz von Notarzthubschraubern [5] oder auch bodengebunden mittels „Mobile Intensive Care Units“ [7] als Unterstützung für die Paramedics zum Einsatz. Als Beispiel sei hier London erwähnt, wo vor mehr als 40 Jahren die Paramedics erfunden wurden und nun seit rund 10 Jahren auch Notärzte nach strengem Indikationsprofil für hochspezifische notärztliche Maßnahmen eingesetzt werden. Demgegenüber erkennen jene Länder, welche seit Jahrzehnten über ein flächendeckendes Notarztsystem verfügen, dass die erreichte Quantität auch zu einem überbordenden Einsatzaufkommen führen kann $[3,4]$.

Das Medizinerkorps Graz stellt zweifellos eine spezifische Einrichtung dar, die sicherlich nicht ohne weiteres auf andere Städte und Versorgungsbereiche übertragbar ist. Jedoch zeigt das dreistufige Modell deutlich auf, dass durch breitere Streuung unterschiedlicher Qualifikationen, orientiert an bestehenden rechtlichen Rahmenbedingungen [12] eine bedarfsadaptierte Optimierung des Notfallrettungswesens möglich ist. Die vorliegende Arbeit will daher keinesfalls ein „Paramedic-System“ befürworten, sondern dem Notarztsystem den ihm zukommenden Stellenwert zurückzugeben: die Versorgung von Notfallpatienten, die kritisch erkrankt bzw. verletzt sind. Selbstredend müssen dazu aber zunächst die tatsächliche Ausbildung der Sanitäter verbessert und bereits vorhandene gesetzliche Möglichkeiten genutzt werden. 


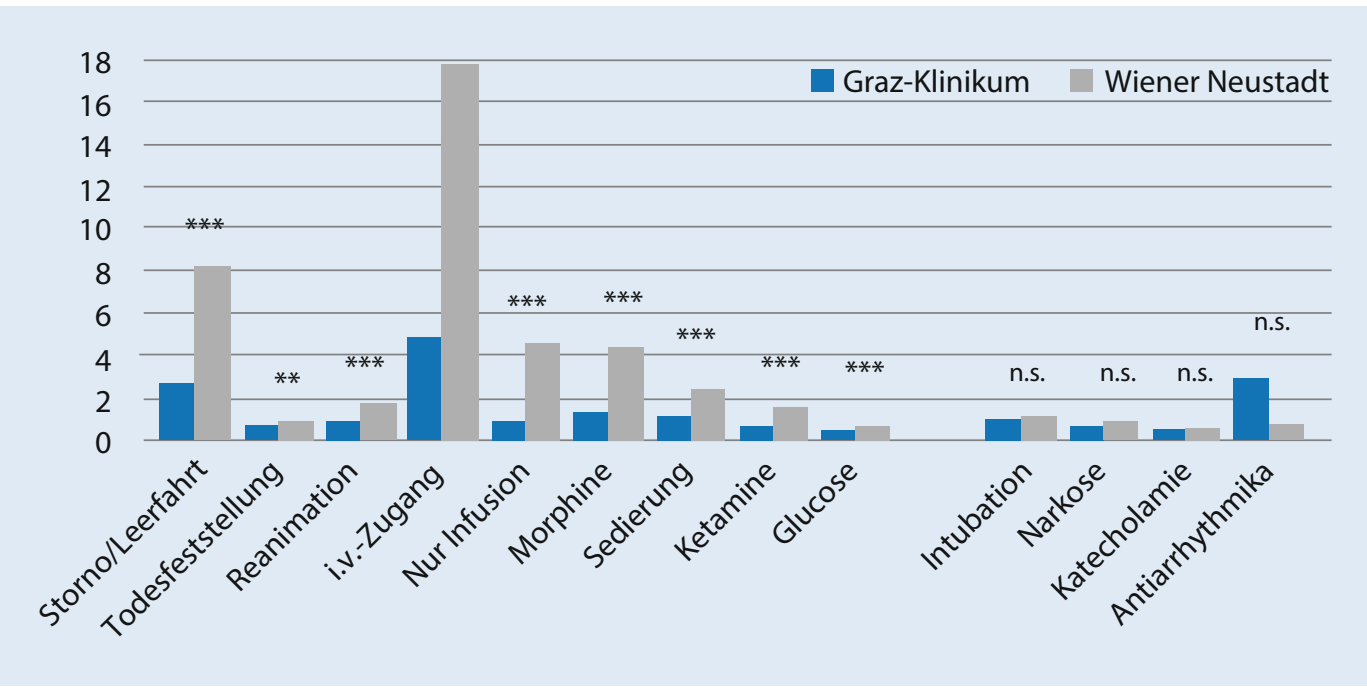

Abb. $2 \triangleleft$ Die notärztlichen Maßnahmen in Bezug auf 1000 Einwohner im Vergleich zwischen den Notarztstützpunkten Graz-Klinikum und Wiener Neustadt $\left({ }^{* * *} p<0,000,{ }^{* *} p<\right.$ 0,01, n. s. nicht signifikant)

\section{Limitationen}

Angesichts der in Österreich noch immer fehlenden einheitlichen Notarztbzw. rettungsdienstlichen Dokumentation kann diese Arbeit nur ein Schlaglicht auf den Status quo und die Entwicklungsmöglichkeiten im Rettungsdienst geben. Einerseits sind Einsatzdaten von manchen Bundesländern nicht exakt verfügbar, die Duplizität von Einsätzen mit teils bodengebundenem Notarztsystem und Hubschraubern kann ebenfalls nicht explizit abgegrenzt werden, da vor allem auch die Randgebiete der Städte von der Flugrettung betreut werden, sodass die Zuordnung zum Einzugsgebiet nicht absolut exakt ist. Nicht zuletzt ändert sich je nach Tages- oder Jahreszeit auch die Bevölkerungszahl aufgrund von Berufsleben und Tourismus, weshalb genaue Versorgungsbereiche und die Umrechnung auf die Einwohnerzahl für ein Notarzteinsatzmittel schwer einzuschätzen sind. Dasselbe gilt auch für die Abgrenzung der Versorgungsbereiche des Notarztsystems Graz, wobei man jedoch davon ausgehen kann, dass der Anteil der „Ausreißer“ sich gegenseitig aufhebt. Für genaue Analysen der notärztlichen Tätigkeiten gibt es von Ausnahmen abgesehen - in Österreich keine vergleichbaren qualifizierten Datenbanken, sodass man sich hier auf die verfügbaren Dokumentationen Graz und Wiener Neustadt fokussieren musste, was nicht zwingend dem öster- reichischen Durchschnitt entsprechen muss.

\section{Fazit für die Praxis}

- Der Wirkungsbereich eines Notarztmittels in Österreich ist je nach Bundesland unterschiedlich und reicht von etwa 1:42.500 EW bis hinauf zu 1:150.000 bzw. im Raum Graz bei 1:200.000 EW.

- Ebenso divergierend ist die Zahl der Notarztanforderungen, die in Österreich durchschnittlich bei $23 / 1000$ liegt, aber von maximal 32 (Wiener Neustadt) bis auf minimal 9 (Graz) reicht.

- Im Gegensatz zum österreichischen Standard verfügt das Notarztsystem Graz über das Medizinercorps. Dabei handelt es sich um Studierende der Humanmedizin ab dem 3. Ausbildungsjahr, die zudem als hochqualifizierte Rettungsmediziner auf Basis des NKI (Notfallsanitäter mit besonderer Notfallkompetenz Intubation) ausgebildet sind und die Notfallrettungswagen des Roten Kreuzes Graz Stadt betreuen. Die zusätzliche Qualifikationsstufe ermöglicht eine vergleichbar geringere Anforderungshäufigkeit der Ressource Notarzt, was nicht zuletzt eine bessere Fokussierung und damit auch deutliche Motivationssteigerung in der Ärzteschaft bewirkt.

- Im Vergleich der Notarztsysteme Graz (3-stufig) und Wiener Neu- stadt (2-stufig) zeigt es sich, dass die hochspezifischen Maßnahmen (Intubation, Narkoseeinleitung usw.) von den Notärzten der beiden Systeme gleich oft vorgenommen werden, während die medizinischen Standardtätigkeiten wie i. v.-Zugang, Infusionstherapie usw. und auch die Fehleinsätze und Stornierungen im 3-stufigen Modell Graz signifikant seltener vorkommen.

\section{Korrespondenzadresse}

Ao. Univ. Prof. Dr. G. Prause

Klinische Abteilung für Allgemeine

Anästhesiologie, Notfall- und Intensivmedizin, Univ. Klinik für Anästhesiologie und Intensivmedizin, LKH Univ. Klinikum Graz Auenbruggerplatz 29, 8036 Graz, Österreich gerhard.prause@medunigraz.at

Open access funding provided by Medical University of Graz.

\section{Einhaltung ethischer Richtlinien}

Interessenkonflikt. G. Prause, G. Wildner, G. Gemes, P. Zoidl, P. Zajic, J. Kainz, M. Pock und H. Trimmel geben an, dass kein Interessenkonflikt besteht.

Dieser Beitrag beinhaltet keine von den Autoren durchgeführten Studien an Menschen oder Tieren.

Open Access. Dieser Artikel wird unter der Creative Commons Namensnennung 4.0 International Lizenz (http://creativecommons.org/licenses/by/4.0/deed. de) veröffentlicht, welche die Nutzung, Vervielfältigung, Bearbeitung, Verbreitung und Wiedergabe in jeglichem Medium und Format erlaubt, sofern Sie den/die ursprünglichen Autor(en) und die Quelle ordnungsgemäßnennen, einen Linkzur Creative Com- 


\section{Originalien}

mons Lizenz beifügen und angeben, ob Änderungen vorgenommen wurden.

\section{Literatur}

1. Bundesministerium der Justiz und für Verbraucherschutz (2016), Deutschland, Gesetz über den Beruf der Notfallsanitäterin und des Notfallsanitäters (Notfallsanitätergesetz - NotSanG) NotSanG. $1-13$.

2. Davis DP, Peay J, Sise MJ et al (2010) Prehospital airway and ventilation management: a trauma score and injury severity score-based analysis. J Trauma 69:294-301. doi:10.1097/TA.0b013e3181dc6c7f

3. Fischer M, Kamp J, Riesgo LG et al (2011)

Comparing emergency medical service systems A project of the European Emergency Data (EED) Project. Resuscitation 82:285-293. doi:10.1016/j. resuscitation.2010.11.001

4. Gries A, Zink W, Bernhard M et al (2006) Realistic assessment of the physician-staffed emergency services in Germany. Anaesthesist 55:1080-1086. doi:10.1007/s00101-006-1051-2

5. Langhelle A, Lossius HM, Silfvast T et al (2004) International EMS systems: the Nordic countries. Resuscitation 61:9-21.doi:10.1016/j.resuscitation. 2003.12.008

6. Lockey DJ (2009) International EMS systems: geographical lottery and diversity but many common challenges. Resuscitation 80:9572. doi:10.1016/j.resuscitation.2009.04.006
7. Papaspyrou E, Setzis D, Grosomanidis V et al (2004) International EMS systems: Greece. Resuscitation 63:255-259. doi:10.1016/j.resuscitation.2004.06. 009

8. Pozner CN, Zane R, Nelson SJ, Levine M (2004) International EMS systems: the United States: past, present, and future. Resuscitation 60:239-244. doi:10.1016/j.resuscitation.2003.11.004

9. Prause G, Kainz J (2014) Entwicklung des Notarztwesens in Österreich - development of the physician staffed prehospital mergency medical service in Austria. Notarzt 30:258-260. doi:10. 1055/s-0034-1387260

10. Prause G, Oswald S, Himler D et al (2013) The medizinercorps graz: a 120-year-old institution of emergency medicine. Prehosp Emerg Care 17:416-420. doi:10.3109/10903127.2013.785622

11. Probst C, Pape H-C, Hildebrand F et al (2009) 30 years of polytrauma care: an analysis of the change in strategies and results of 4849 cases treated at a single institution. Injury 40:77-83. doi:10.1016/j. injury.2008.10.004

12. Republik Österreich (2002) Bundesgesetz über Ausbildung, Tätigkeiten und Beruf der Sanitäter. Bundesgesetzblatt :139-160; Teil I; Ausgegeben 18. Januar 2002

13. Roessler M, Zuzan O (2006) EMS systems in Germany. Resuscitation 68:45-49. doi:10.1016/j. resuscitation.2005.08.004

14. SIRMED:Berufsbildungen im Rettungsdienst. 2016 Schweizer Institut für Rettungsmedizin 1-10.

15. Trimmel H, Wodak A, Voelckel W (2006) Hubschrauberdisposition mit dem Advanced-Medical-
Priority-Dispatch-System - Erwartungen erfüllt? Notf Rettungsmed 9:437-445. doi:10.1007/ s10049-006-0837-8

16. Wikipedia (2016) Rettungsfachpersonal. https:// de.wikipedia.org/wiki/Rettungsfachpersonal. Zugegriffen:25. Januar 2017

\section{Hier steht eine Anzeige.}

\section{Springer}

